Supporting Information

\title{
Atomic Model for Alkali Metal Passivation of Point Defects at Perovskite Grain Boundaries
}

\author{
Lu Qiao ${ }^{1}$, Wei-Hai Fang ${ }^{1}$, Run Long ${ }^{1 *}$, Oleg V. Prezhdo ${ }^{2}$ \\ ${ }^{1}$ College of Chemistry, Key Laboratory of Theoretical \& Computational Photochemistry of \\ Ministry of Education, Beijing Normal University, Beijing, 100875, People's Republic of \\ China \\ ${ }^{2}$ Department of Chemistry, University of Southern California, Los Angeles, CA 90089, United
} States

\section{Simulation Details}

Geometry optimization, adiabatic MD, and NA coupling calculations are carried out at the spin-non-polarized DFT level using the Vienna ab initio Simulation Package (VASP). ${ }^{1}$ Spinpolarized calculations are performed to confirm the location of the trap states in the oddelectron systems, as reported in Supporting Information (SI). The Perdew-Burke-Ernzerhof (PBE) functional ${ }^{2}$ is used to describe the electron exchange-correlation effects. The projectedaugmented wave method is used to describe interactions between electrons and ion cores, ${ }^{3}$ with a plane wave energy cutoff of $400 \mathrm{eV}$. The Grimme's DFT-D3 correction is employed to describe the van der Waals interactions. ${ }^{4}$ Spin-orbit coupling (SOC) is important in $\mathrm{MAPbI}_{3}$ due to the presence of heavy lead and iodine atoms. The combination of PBE and SOC significantly underestimates the bandgap of $\mathrm{MAPbI}_{3},{ }^{5}$ while the PBE functional produces good values for the bandgap ${ }^{6,7}$ due to cancellation of the SOC contribution and the self-interaction error. ${ }^{8}$ In particular, the PBE-SOC bandgap value of $0.56 \mathrm{eV}^{9}$ is nearly three times smaller than the experimental value of $1.52 \mathrm{eV} \cdot{ }^{10}$ Hybrid functionals and the $\mathrm{GW}$ approach notably overestimate the bandgap of $\mathrm{MAPbI}_{3}$ and must be combined with SOC to generate good

\footnotetext{
*Corresponding author, E-mail: runlong@bnu.edu.cn
} 
values. ${ }^{11,12}$ However, such levels of electronic structure theory cannot be used in the current simulation, because NAMD requires thousands of electronic structure calculations to produce NA couplings and generate an ensemble of quantum dynamics trajectories. It is important to use correct energy gap values when studying charge trapping and recombination. We use PBE without SOC, because it gives much better agreement with experiment than PBE with SOC. The approach allowed us to obtain good results while studying photoinduced dynamics in $\mathrm{MAPbI}_{3}$ containing dopants ${ }^{13}$ and grain boundaries ${ }^{6}$, localized charges ${ }^{14}$, and interfaced with water ${ }^{15}$ and $\mathrm{TiO}_{2} \cdot{ }^{16}$

The 240 -atom $2 \times 2 \times 5$ pristine $\mathrm{MAPbI}_{3}$ supercell and $\Sigma 5(310) \mathrm{GBs}$ are used to construct the MA $A_{\mathrm{Pb}} @$ pristine, MA $\mathrm{Pb} @ \mathrm{~GB}$ and K/Rb passivated MA $\mathrm{Pb}_{\mathrm{Pb}} @ \mathrm{~GB}$ systems. The structures are optimized by employing the $3 \times 3 \times 1$ and $6 \times 2 \times 1$ Monkhorst-Pack k-point meshes ${ }^{17}$ for the pristine and GB related systems, respectively. After geometry optimization, all systems are heated to $300 \mathrm{~K}$ by repeated velocity rescaling for $2 \mathrm{ps}$. Then, 5 ps adiabatic MD trajectories are generated in the microcanonical ensemble with a $1 \mathrm{fs}$ atomic time step. Due to the limited duration of the MD trajectories and NA couplings, compared to the charge trapping and recombination times, we perform short-time NAMD simulations to obtain the transition rates for each pair of electronic states, by fitting the data using an exponential function. After that, we solve the coupled kinetic equations presented in SI (Figures S1-S2). The short-time NAMD simulations are carried out using the PYthon eXtension for Ab Initio Dynamics (PYXAID) code, ${ }^{18,19}$ in which all 5000 geometries from the 5 ps MD trajectories are employed as initial conditions, and 1000 random number sequences are used for each geometry. The simulated population decay is shown in Figures S3-S5. Additional computational details can be found in our previous work. ${ }^{20}$

We chose a $2 \times 2 \times 5$ supercell for pristine $\mathrm{MAPbI}_{3}$ in order to keep the same number atoms in both pristine and $\Sigma 5(310)$ GB systems. Such consistency is important for direct comparison of NAMD simulations because the magnitude of NA coupling depends on phonon modes available in the system. MA cations spontaneously rotate at ambient conditions, as demonstrated by various MD simulations including our own work. ${ }^{13,21}$ 


\section{Defect Formation Energies}

The defect formation energies are calculated by the following general equation:

$$
E_{\text {form }}=E_{\text {defect }}-E_{\text {perfect }}+\text { a } E_{i}
$$

$E_{\text {defect }}$ and $E_{\text {perfect }}$ represent the total energy of the GB system with and without a defect, $E_{\mathrm{i}}$ represents the energy of a single atom or a molecule. $a=-1$ corresponds to the molecule or atom introduced into the system, while $a=1$ corresponds to the molecule or atom removed from the system. For example, the formation energy of $\mathrm{MA}_{\mathrm{Pb}}$ is obtained by: $E_{M A P b}=E_{M A P b} @ G B$ $-E_{G B}-E_{M A}+E_{P b}$

Table S1. The formation energies of ten defects in $\mathrm{GB}$, including antisites $\left(\mathrm{MA}_{\mathrm{Pb}}, \mathrm{Pb}_{\mathrm{MA}}, \mathrm{I}_{\mathrm{Pb}}\right.$, $\left.\mathrm{Pb}_{\mathrm{I}}\right)$, interstitials $\left(\mathrm{MA}_{\mathrm{i}}, \mathrm{Pb}_{\mathrm{i}}, \mathrm{I}_{\mathrm{i}}\right)$ and vacancies $\left(\mathrm{V}_{\mathrm{MA}}, \mathrm{V}_{\mathrm{Pb}}, \mathrm{V}_{\mathrm{I}}\right)$.

\begin{tabular}{|c|c|c|c|c|c|c|c|c|c|c|}
\hline & $\mathrm{MA}_{\mathrm{Pb}}$ & $\mathrm{Pb}_{\mathrm{MA}}$ & $\mathrm{I}_{\mathrm{Pb}}$ & $\mathrm{Pb}_{\mathrm{I}}$ & $\mathrm{MA}_{\mathrm{i}}$ & $\mathrm{Pb}_{\mathrm{i}}$ & $\mathrm{I}_{\mathrm{i}}$ & $\mathrm{V}_{\mathrm{MA}}$ & $\mathrm{V}_{\mathrm{Pb}}$ & $\mathrm{V}_{\mathrm{I}}$ \\
\hline$(\mathrm{eV})$ & -0.765 & 5.286 & -0.617 & 5.426 & -0.939 & 6.550 & 0.455 & 3.187 & 0.236 & 1.996 \\
\hline
\end{tabular}

\section{Kinetics Models}

The schematics and basis set of the charge relaxation processes for the pristine $\mathrm{MAPbI}_{3}$, $\mathrm{MAPbI}_{3}$ with GBs, MA $\mathrm{Pb}_{0} @$ pristine, KMA $\mathrm{Pb}_{\mathrm{Pb}} @ \mathrm{~GB}$ and RbMA $\mathrm{Pb}_{\mathrm{P}} @ \mathrm{~GB}$ systems are depicted in Figure S1. The corresponding information for the $\mathrm{MA}_{\mathrm{Pb}} @ \mathrm{~GB}$ system is shown in Figure $\mathrm{S} 2$. The population decay of the excited state (conduction band minimum, CBM) and electron trap state during charge trapping and recombination processes are shown in Figure S3-S5. Fitting the data using the short-time linear approximation of the exponential function, $P(t)=\exp$ $\left(-\frac{t}{\tau}\right) \approx 1-t / \tau$, gives the transition rates $\mathrm{k}_{(\mathrm{cbm} \rightarrow \mathrm{vbm})}, \mathrm{k}_{(\mathrm{cbm} \rightarrow \operatorname{trap})}$ and $\mathrm{k}_{(\mathrm{vbm} \rightarrow \text { trap })}$ between pairs of electronic states. The ground state (VBM), excited state (CBM) and electron trap state are defined as [GS], [ES] and [trap], respectively. 
Pristine $\mathrm{MAPbI}_{3}, \mathbf{G B}, \mathrm{MA}_{\mathrm{Pb}} @$ pristine, KMA $\mathrm{Ab}_{\mathrm{Pb}} @ \mathbf{G B}$, and RbMA $\mathrm{Pb}_{\mathrm{Pb}} @ \mathbf{G B}$ : Electron-hole recombination occurs between the CBM and VBM. Figure S1 presents the basis set. The timeevolution population for the CBM and VBM are described by equations S1 and S2, and their solutions are shown in equations S3 and S4. Figures S3, S4a and S5 show the excited-state population decay and the corresponding rate constants for the five systems.

Coupled kinetic equations for electron-hole recombination:

$$
\begin{aligned}
\frac{\mathrm{d}[\mathrm{ES}]}{\mathrm{dt}} & =-\mathrm{k}_{(\mathrm{cbm} \rightarrow \mathrm{vbm})}[\mathrm{ES}] \\
\frac{\mathrm{d}[\mathrm{GS}]}{\mathrm{dt}} & =\mathrm{k}_{(\mathrm{cbm} \rightarrow \mathrm{vbm})}[\mathrm{ES}]
\end{aligned}
$$

Solutions of above equations:

$$
\begin{aligned}
{[\mathrm{ES}] } & =\mathrm{e}^{-\mathrm{k}_{(\mathrm{cbm} \rightarrow \mathrm{vbm})} * \mathrm{t}} \\
{[\mathrm{GS}] } & =1-\mathrm{e}^{-\mathrm{k}_{(\mathrm{cbm} \rightarrow \mathrm{vbm})} * \mathrm{t}}
\end{aligned}
$$

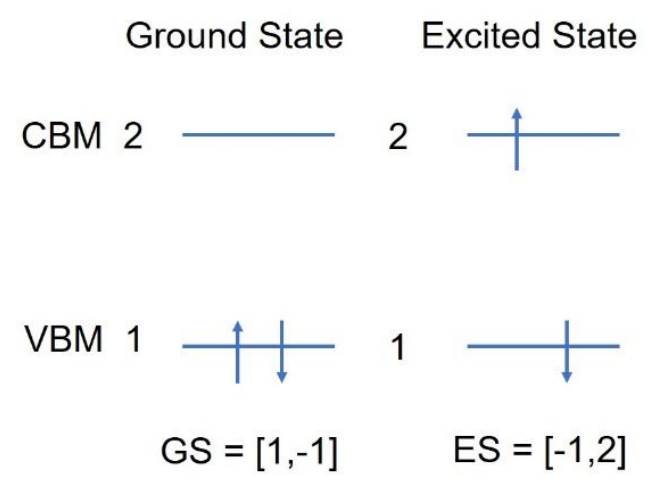

Figure S1. The schematic of electron-hole recombination across the CBM-VBM energy gap in the pristine $\mathrm{MAPbI}_{3}, \mathrm{~GB}, \mathrm{MA}_{\mathrm{Pb}} @$ pristine, KMA $\mathrm{Pb} @ \mathrm{~GB}, \mathrm{RbMA} \mathrm{Pb}_{0} @ \mathrm{~GB}$ systems.

MA $\mathbf{P b} @$ GB: Recombination of electron-hole mediated by an electron trap is described by equations S5-S7, and their solutions are presented in equations S8-S10. Figure S2 displays the basis set. Figures S4b-4d show the population decay of the key states and the corresponding rate constants.

Coupled kinetic equations for the electron trap assisted electron-hole recombination:

$$
\frac{\mathrm{d}[\mathrm{ES}]}{\mathrm{dt}}=-\left(\mathrm{k}_{(\mathrm{cbm} \rightarrow \mathrm{vbm})}+\mathrm{k}_{(\mathrm{cbm} \rightarrow \operatorname{trap})}\right)[\mathrm{ES}]
$$




$$
\begin{aligned}
& \frac{\mathrm{d}[\text { trap }]}{\mathrm{dt}}=\mathrm{k}_{(\mathrm{cbm} \rightarrow \operatorname{trap})}[\mathrm{ES}]-\mathrm{k}_{(\operatorname{trap} \rightarrow \mathrm{vbm})}[\operatorname{trap}] \\
& \frac{\mathrm{d}[\mathrm{GS}]}{\mathrm{dt}}=\mathrm{k}_{(\mathrm{cbm} \rightarrow \mathrm{vbm})}[\mathrm{ES}]+\mathrm{k}_{(\operatorname{trap} \rightarrow \mathrm{vbm})}[\operatorname{trap}]
\end{aligned}
$$

\section{Solutions of above equations:}

$$
\begin{aligned}
& {[E S]=\mathrm{e}^{-\left(\mathrm{k}_{(\mathrm{cbm} \rightarrow \mathrm{vbm})}+\mathrm{k}_{(\mathrm{cbm} \rightarrow \mathrm{trap})}\right) * \mathrm{t}}} \\
& {[\operatorname{trap}]=\frac{\mathrm{k}_{(\mathrm{cbm} \rightarrow \mathrm{trap})}}{\left(\mathrm{k}_{(\mathrm{cbm} \rightarrow \mathrm{vbm})}+\mathrm{k}_{(\mathrm{cbm} \rightarrow \mathrm{trap})}-\mathrm{k}_{(\mathrm{trap} \rightarrow \mathrm{vbm})}\right)}} \\
& \left(\mathrm{e}^{-\mathrm{k}_{(\text {trap } \rightarrow \mathrm{vbm})} * \mathrm{t}}-\quad \mathrm{e}^{-\left(\mathrm{k}_{(\mathrm{cbm} \rightarrow \mathrm{vbm})}+\mathrm{k}_{(\mathrm{cbm} \rightarrow \mathrm{trap})}\right) * \mathrm{t}}\right) \\
& {[\mathrm{GS}]=1-\frac{1}{\left(\mathrm{k}_{(\mathrm{cbm} \rightarrow \mathrm{vbm})}+\mathrm{k}_{(\mathrm{cbm} \rightarrow \text { trap })}-\mathrm{k}_{(\text {trap } \rightarrow \mathrm{vbm})}\right)}\left\{\mathrm{k}_{(\mathrm{cbm} \rightarrow \text { trap })}\right.} \\
& \left.* \mathrm{e}^{-\mathrm{k}_{(\mathrm{trap} \rightarrow \mathrm{vbm})} * \mathrm{t}}-\left(\mathrm{k}_{(\mathrm{cbm} \rightarrow \mathrm{vbm})}-\mathrm{k}_{(\mathrm{trap} \rightarrow \mathrm{vbm})}\right) * \mathrm{e}^{-\left(\mathrm{k}_{(\mathrm{cbm} \rightarrow \mathrm{vbm})}+\mathrm{k}_{(\mathrm{cbm} \rightarrow \mathrm{trap})}\right) * \mathrm{t}}\right\}
\end{aligned}
$$

\section{Ground State Electron Trap Excited State}

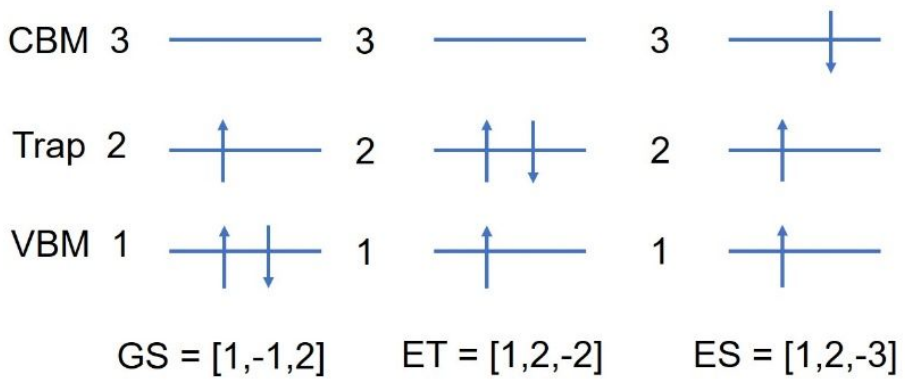

Figure S2. The schematic of the electron trap assisted electron-hole recombination in the MAPb $@ G B$ system.
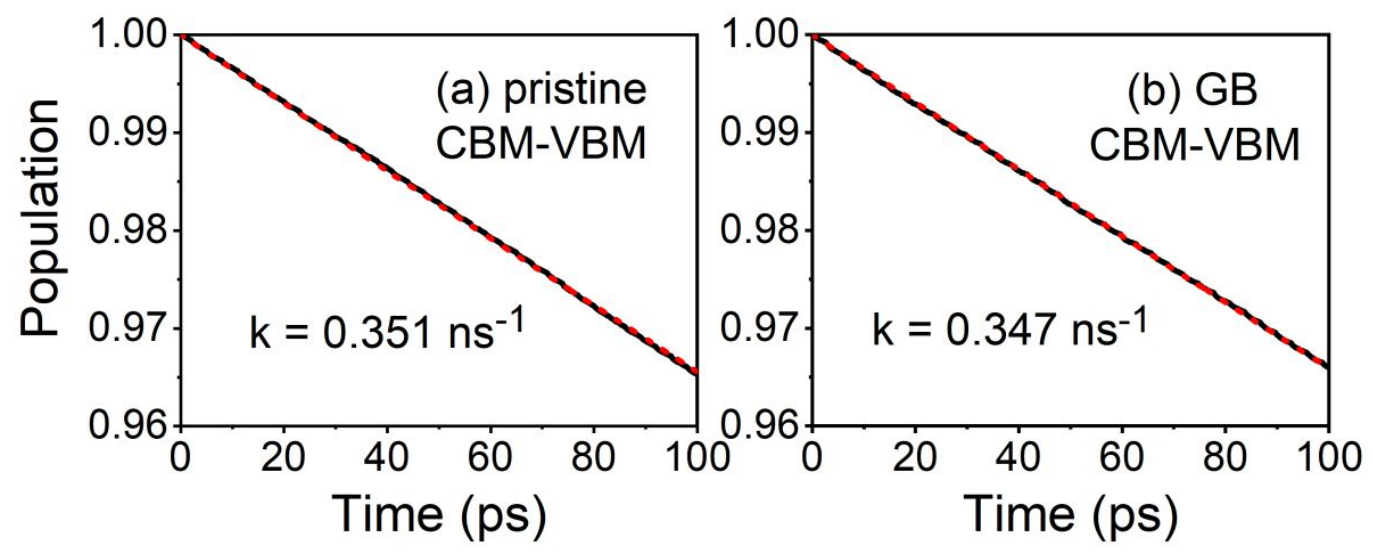

Figure S3. Decay of population of the excited-state during transition from CBM to VBM in (a) pristine $\mathrm{MAPbI}_{3}$ and (b) GB systems. 

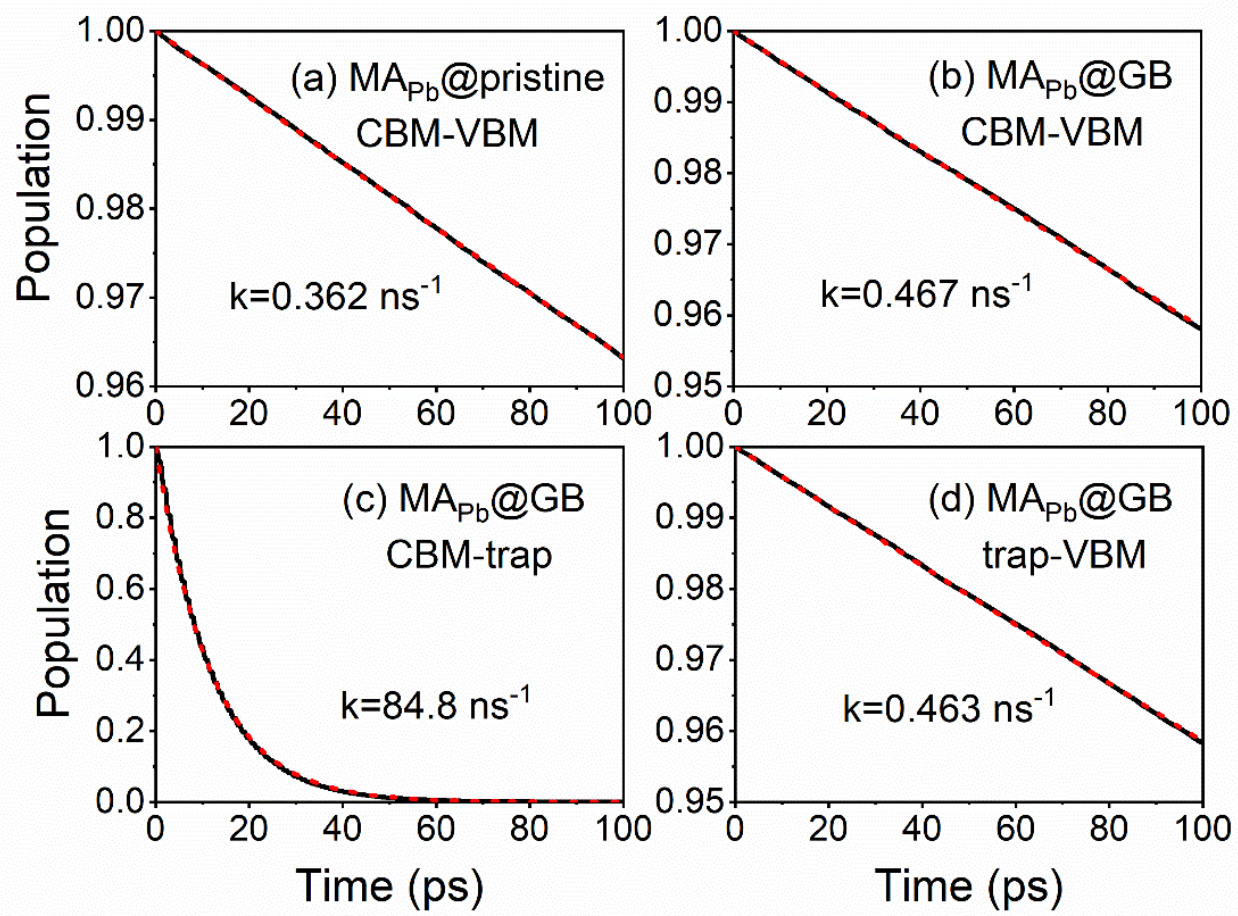

Figure S4. Decay of population of the excited-state during transition from CBM to VBM in (a) $\mathrm{MA}_{\mathrm{Pb}} @$ pristine. $\mathrm{CBM}$ population decay during transition from CBM to either (b) VBM or trap (c) in $\mathrm{MA}_{\mathrm{Pb}} @ \mathrm{~GB}$. (d) Trap state population decay during transition from electron trap to VBM in MAPb@GB.
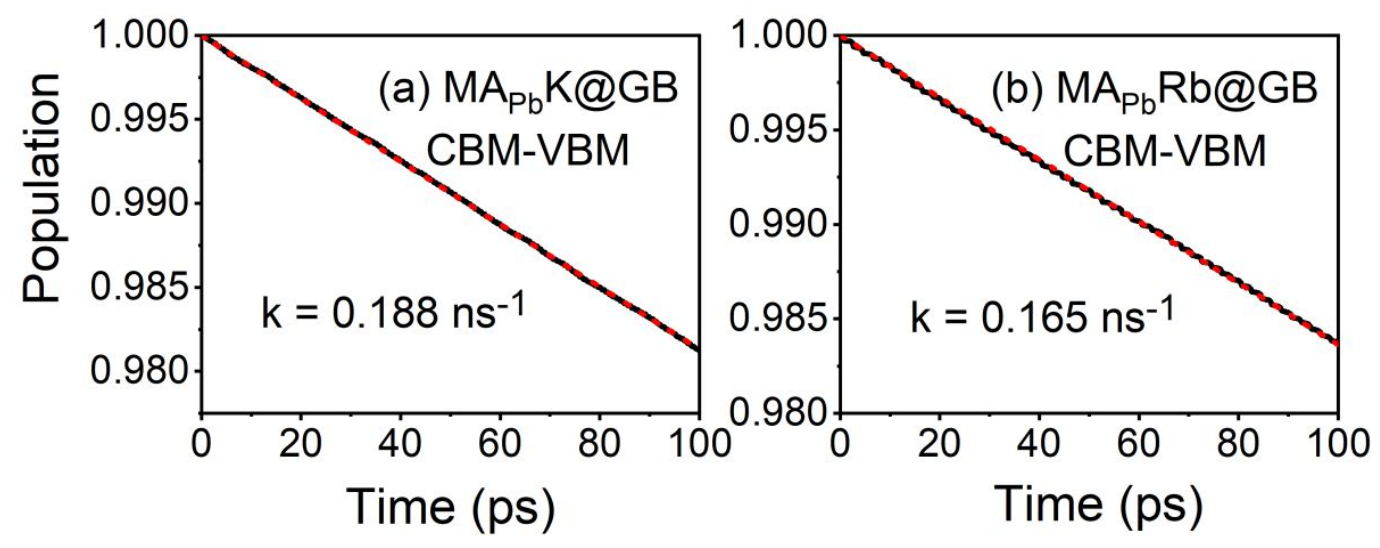

Figure S5. Decay of population of the excited-state during transition from CBM to VBM in (a) KMA $\mathrm{Pb}_{0} @ \mathrm{~GB}$ and (b) RbMA $\mathrm{Pb} @ \mathrm{~GB}$. 
(a) pristine

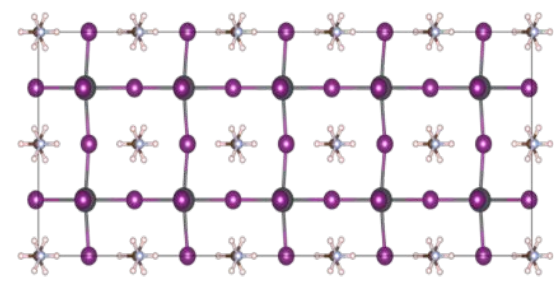

\section{$\circ \circ \circ \circ 0$ \\ $\mathrm{HCN}$ I $\mathrm{Pb}$}

(b) GB

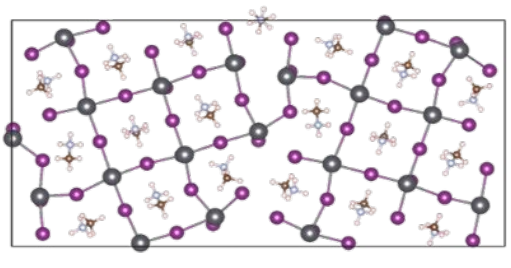
$\mathrm{Pb}$

Figure S6. Optimized structures of (a) $2 \times 2 \times 5$ 240-atom pristine $\mathrm{MAPbI}_{3}$, and (b) 240atom $\Sigma 5(310) \mathrm{GB}$.

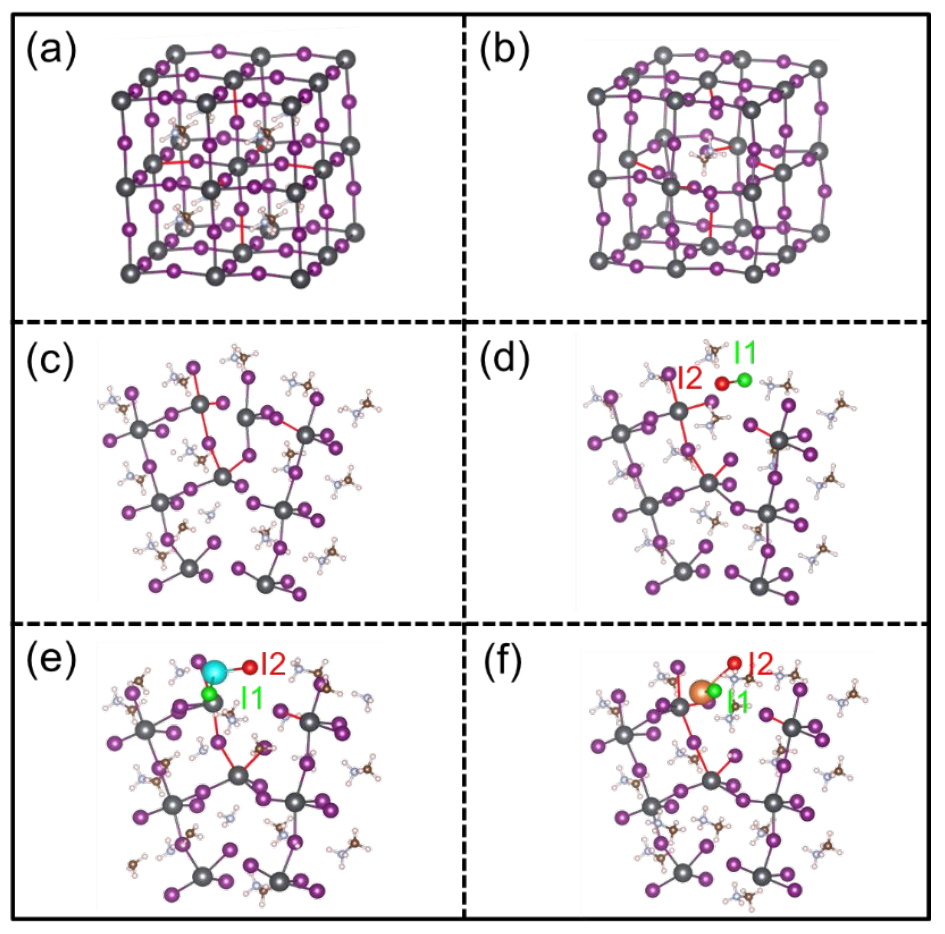

Figure S7. Magnified local strtcure of (a) pristine $\mathrm{MAPbI}_{3}$, (b) $\mathrm{MA}_{\mathrm{Pb}} @$ pristine, (c) GB, (d) $\mathrm{MA}_{\mathrm{Pb}} @ \mathrm{~GB},(\mathbf{e}) \mathrm{KMA}_{\mathrm{Pb}} @ \mathrm{~GB}$ and (f) RbMA $\mathrm{Pb} @ \mathrm{~GB}$. The structures in parts c-d are rotated by $40^{\circ}$ outside the plane to show the I-dimer clearly. The six $\mathrm{Pb}$-I bonds around $\mathrm{MA} \mathrm{Pb}_{\mathrm{Pb}}$ are marked by red lines. The I-dimer forms in $\mathrm{MA}_{\mathrm{Pb}} @ \mathrm{~GB}$ between the I1 and I2 atoms. The dimer is broken in the KMA $\mathrm{Pb}_{\mathrm{Pb}} @ \mathrm{~GB}$ and RbMA $\mathrm{Pb} @ \mathrm{~GB}$ systems. 


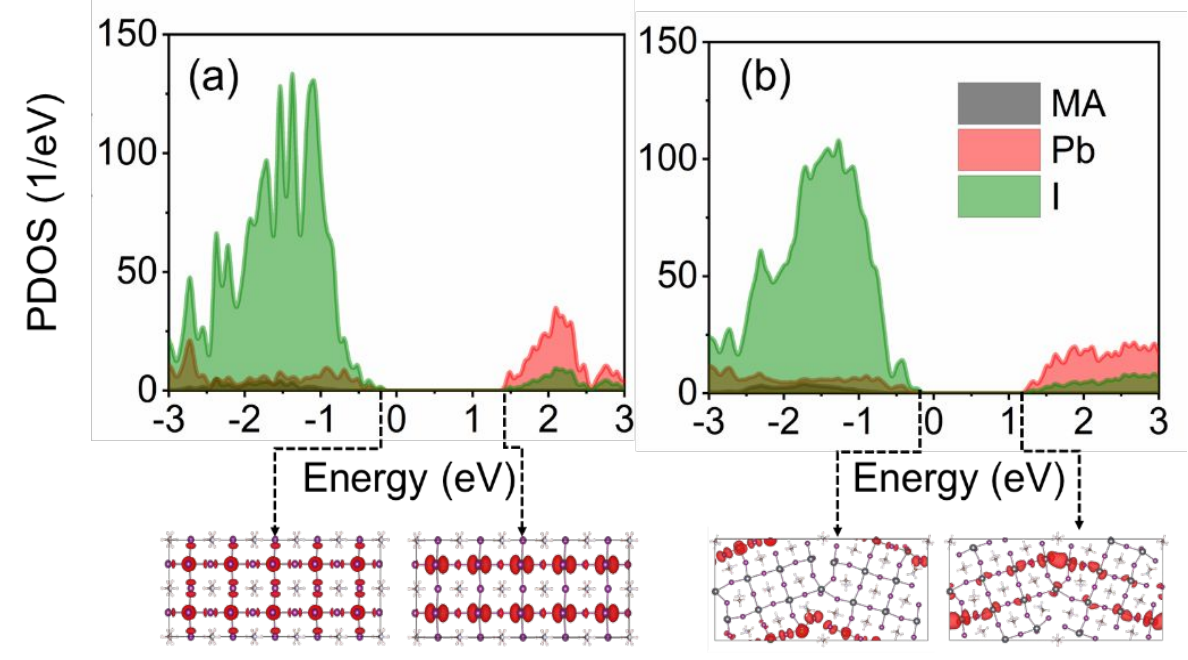

Figure S8. Projected densities of states (PDOS) (top panels) and charge densities (bottom panels) of CBM and VBM for (a) pristine $\mathrm{MAPbI}_{3}$ and (b) GB.
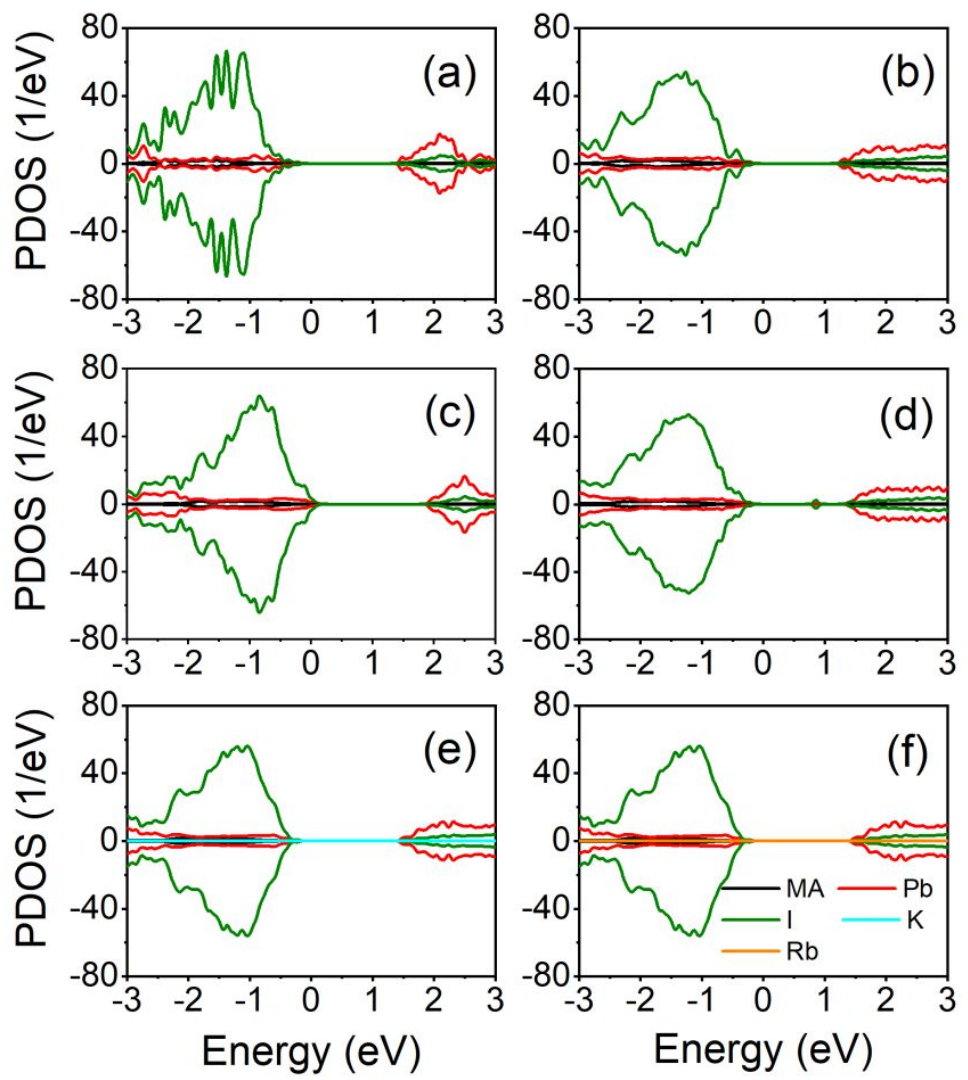

Figure S9. Spin-polarized PDOS of (a) pristine $\mathrm{MAPbI}_{3}$, (b) GB, (c) $\mathrm{MA}_{\mathrm{Pb}} @$ pristine, (d) MA $A_{\mathrm{Pb}} @ G B,(\mathbf{e}) \mathrm{KMA}_{\mathrm{Pb}} @ \mathrm{~GB}$, and (f) RbMA $\mathrm{Pb} @ \mathrm{~GB}$ calculated at the PBE level. 

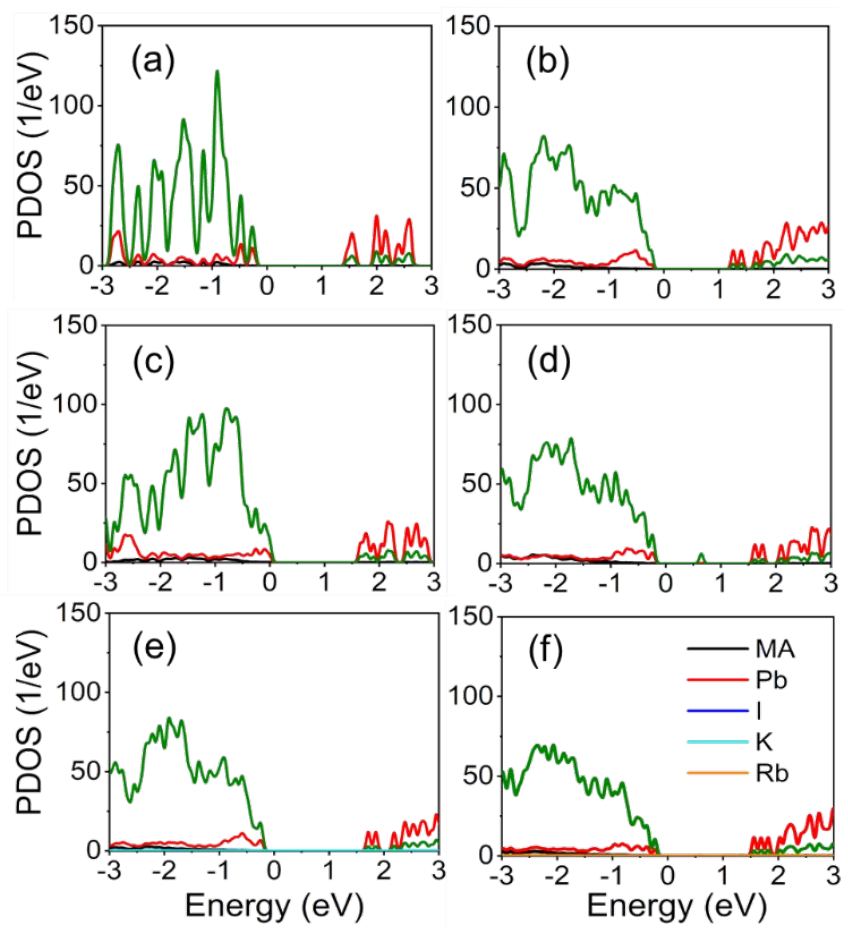

Figure S10. PDOS of (a) pristine $\mathrm{MAPbI}_{3}$, (b) GB (c) MA $\mathrm{Pb}_{\mathrm{Pb}} @$ pristine, (d) $\mathrm{MA}_{\mathrm{Pb}} @ \mathrm{~GB}$, (e) $\mathrm{KMA}_{\mathrm{Pb}} @ \mathrm{~GB}$, and (f) RbMA $\mathrm{Pb} @ \mathrm{~GB}$ calculated at the HSE06+SOC level and at the single kpoint corresponding to the bandgap, due to computational cost.

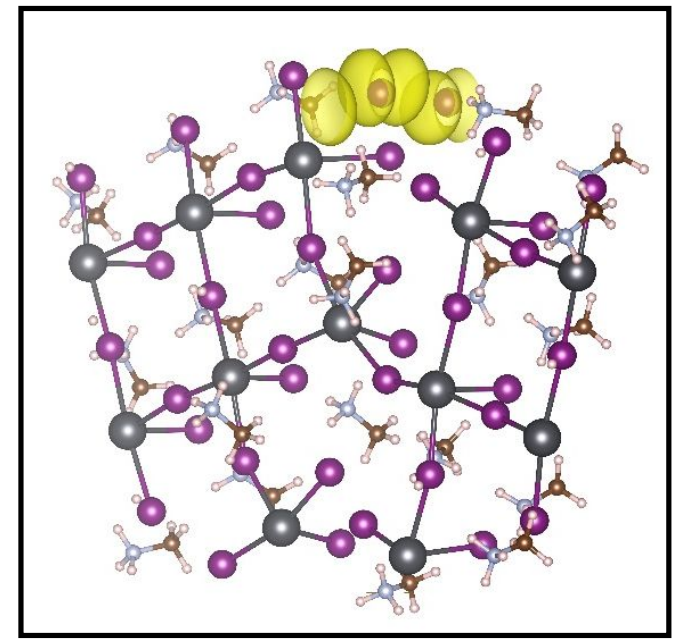

Figure S11. Amplified charge density of the trap state in MA $A_{\mathrm{Pb}} @ \mathrm{~GB}$. The charge concentrates on the I-dimer. 


\section{Pure-Dephasing Functions}

The pure-dephasing functions characterize elastic electron-vibrational interactions that are responsible for coherence loss in the electronic subsystem. Figure S12 displays the puredephasing functions of MA $\mathrm{Pb}_{\mathrm{Pb}} @$ pristine, MA $\mathrm{Pb} @ \mathrm{~GB}, \mathrm{KMA} \mathrm{Pb}_{0} @ \mathrm{~GB}$ and RbMA $\mathrm{Pb} @ \mathrm{~GB}$, computed using the second-order cumulant approximation in the optical response theory. ${ }^{22}$ The pure-dephasing functions of the pristine $\mathrm{MAPbI}_{3}$ and GB systems are shown in Figure S13. Fitting the functions to a Gaussian, $\exp \left(-0.5(\mathrm{t} / \tau)^{2}\right)$, gives the pure-dephasing times, $\tau$ (Table 1 of the main text). The pure-dephasing times for the pristine $\mathrm{MAPbI}_{3}$ and $\mathrm{GBs}$ systems are listed in the parentheses in Table 1. The presence of multiple phonon modes in the influence spectra leads to fast sub-10 fs pure-dephasing. Short pure-dephasing times favor slow quantum transitions. Quantum transitions stop when the pure-dephasing time becomes infinitesimal, as exemplified by the quantum Zeno effect. ${ }^{23}$ As seen in Table 1, the shorter pure-dephasing times observed in the $\mathrm{K}$ - and $\mathrm{Rb}$-passiviated $\mathrm{MA}_{\mathrm{Pb}} @ \mathrm{~GB}$ systems reduce the nonradiative electronhole recombination and further prolong charge carrier lifetimes.

To analyze the pure-dephasing times, we plotted the unnormalized autocorrelation functions (un-ACF) in the insets of Figure S12. In general, a larger initial value of un-ACF, $C_{i j}$ $(t)=\left\langle\delta E_{i j}\left(t^{\prime}\right) \delta E_{i j}\left(t-t^{\prime}\right)\right\rangle_{t^{\prime}}$, leads to faster dephasing, as follows rom the second-order cumulant approximation, $D_{i j}(t)=\exp \left(-\frac{1}{\hbar^{2}} \int_{0}^{t} \mathbb{d} t^{\prime} \int_{0}^{t^{\prime}} \mathbb{d} t^{\prime \prime} C_{i j}\left(t^{\prime \prime}\right)\right) \cdot{ }^{22}$ The initial value, $C_{i j}(0)$ $=\delta E_{i j}^{2}(0)$, represents the square energy gap fluctuation and enters the pure-dephasing function, $D_{i j}(t)$, as a constant in the exponent. The initial values of un-ACF for each pair of electronic states for all the systems are listed in Table 1, rationalizing why the pure-dephasing times for the CBM-VBM transition follow the sequence: $\mathrm{MA} \mathrm{Pb}_{\mathrm{Ob}} \mathrm{GB}$ pristine $\mathrm{MAPbI}_{3}>$ MA $A_{\mathrm{Pb}} @$ prisitne > GB > KMA $\mathrm{Pb} @ \mathrm{~GB}>$ RbMA $\mathrm{Pb}_{\mathrm{P}} @ \mathrm{~GB}$. In the MA $\mathrm{Pb}_{\mathrm{Pb}} @ \mathrm{~GB}$ system, the puredephasing times decay as CBM-VBM > VBM-trap > CBM-trap. The energy gap between the initial and final states influences both the pure-dephasing time and the NA coupling. The gap also enters the time-dependent Schrödinger equation directly, and therefore, it influences charge carrier dynamics in multiple ways. 

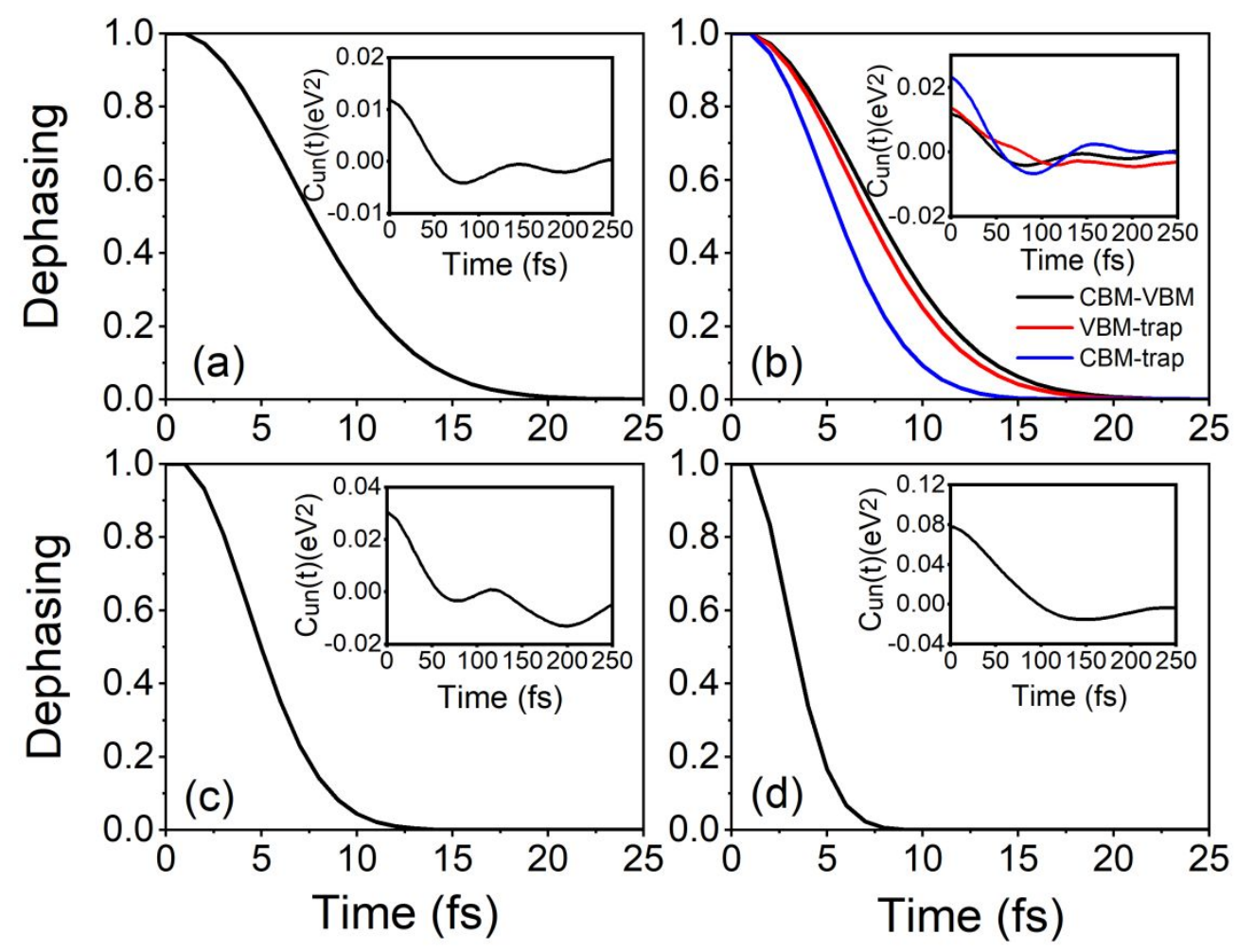

Figure S12. Pure-dephasing functions for the electronic transitions in (a) $\mathrm{MA}_{\mathrm{Pb}} @$ pristine, (b) MA $A_{\mathrm{Pb}} @ \mathrm{~GB}$, (c) KMA $\mathrm{Pb}_{\mathrm{Pb}} @ \mathrm{~GB}$ and (d) RbMA $\mathrm{Pb} @ \mathrm{~GB}$. The insets show the unnormalized autocorrelation functions (un-ACF) of the four systems. The initial value of the un-ACF is large, and pure-dephasing is fast. 


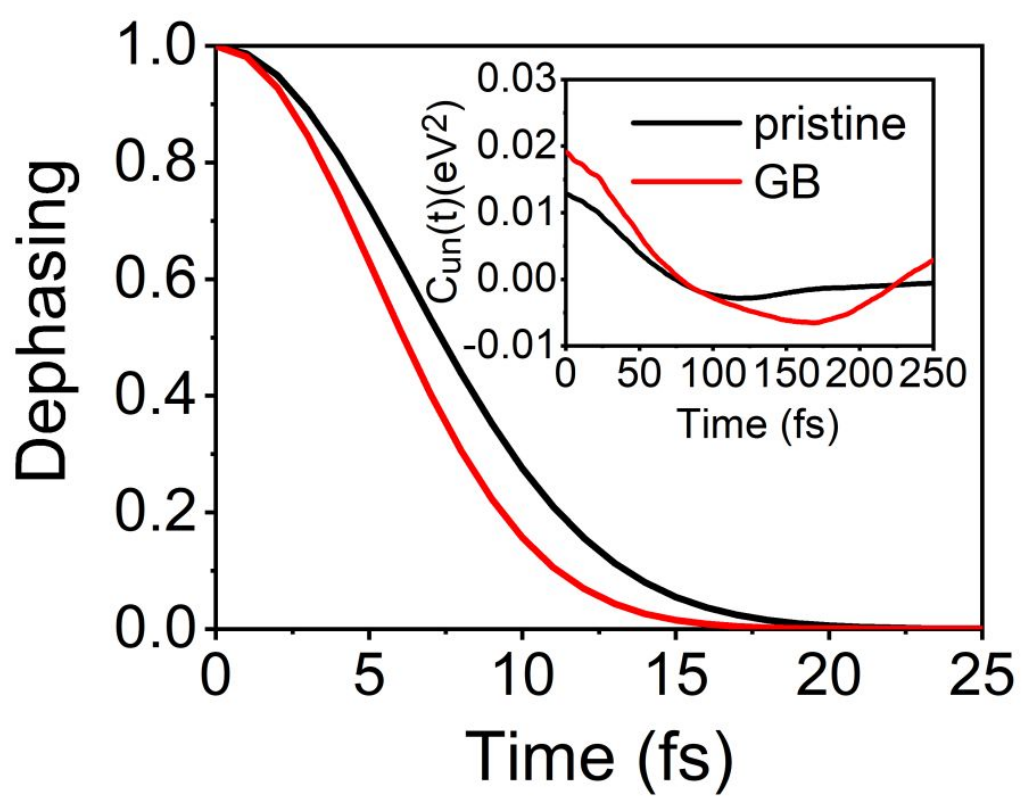

Figure S13. Pure-dephasing functions for the CBM-VBM transition in the pristine $\mathrm{MAPbI}_{3}$ and GB systems. The inset shows the unnormalized autocorrelation functions.

6. Additional Details of Charge Recombination Dynamics

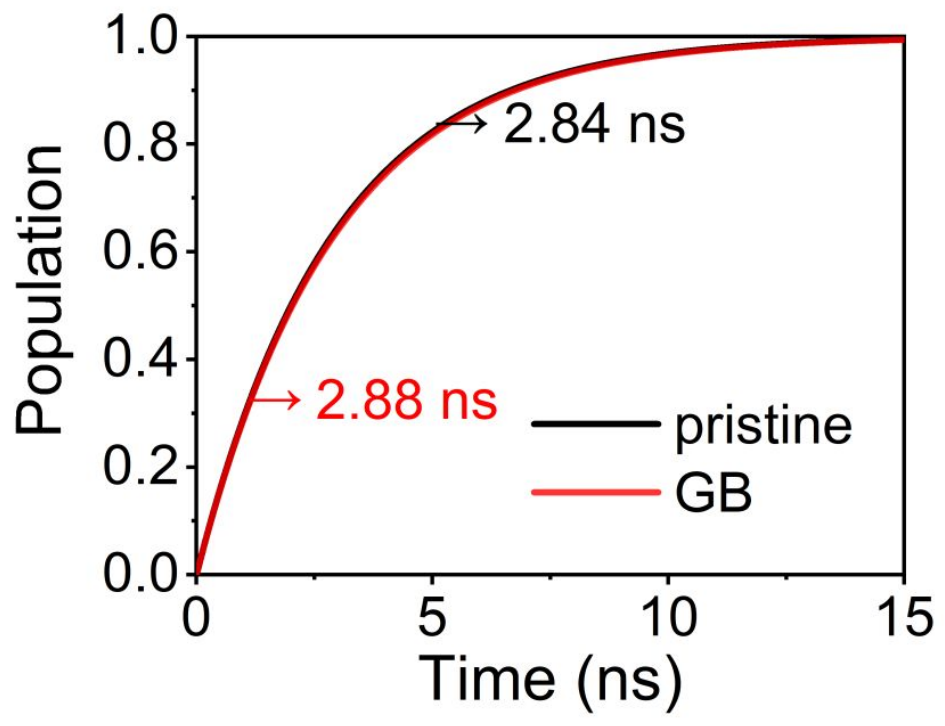

Figure S14. Nonradiative electron-hole recombination dynamics in the pristine $\mathrm{MAPbI}_{3}$ and GB systems. GB has little influence on the recombination time scale. 


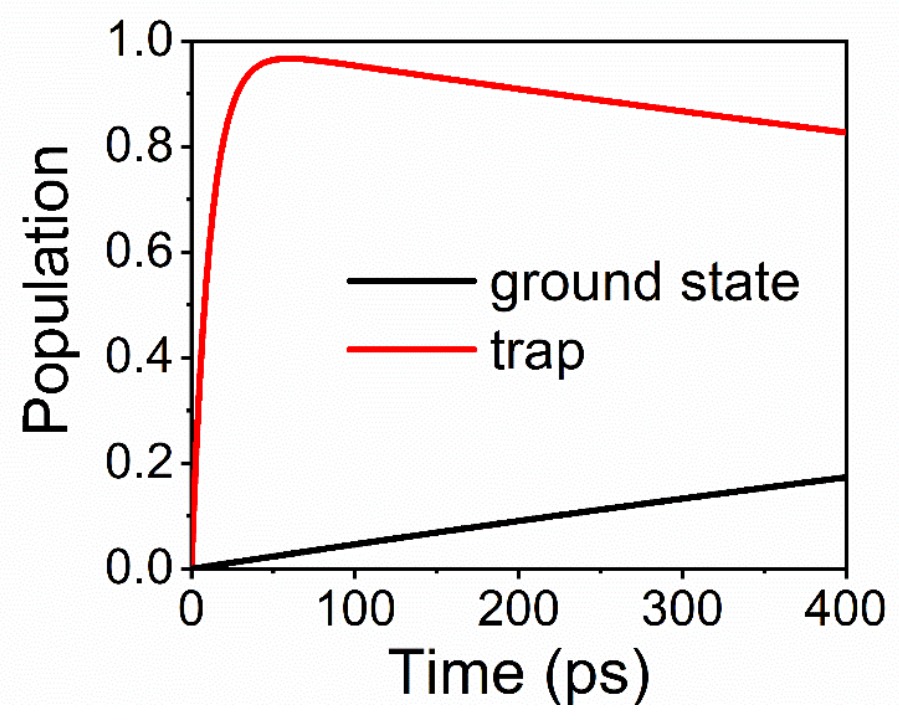

Figure S15. Enlarged graph of the rise of the trap state population in MA $\mathrm{Pb} @ \mathrm{~GB}$.

\section{References}

(1) Kresse, G.; Furthmuller, J., Efficient Iterative Schemes for Ab Initio Total-Energy Calculations Using a Plane-Wave Basis Set. Phys. Rev. B 1996, 54, 11169-11186.

(2) Perdew, J. P.; Burke, K.; Ernzerhof, M., Generalized Gradient Approximation Made Simple. Phys. Rev. Lett. 1996, 77, 3865-3868.

(3) Blochl, P. E., Projector Augmented-Wave Method. Phys. Rev. B: Condens. Matter Mater. Phys. 1994, 50, 17953-17979.

(4) Grimme, S.; Antony, J.; Ehrlich, S.; Krieg, H., A Consistent and Accurate Ab Initio Parametrization of Density Functional Dispersion Correction (Dft-D) for the 94 Elements HPu. J. Chem. Phys 2010, 132, 154104.

(5) Shan, W.; Saidi, W. A., Segregation of Native Defects to the Grain Boundaries in Methylammonium Lead Iodide Perovskite. J. Phys. Chem. Lett. 2017, 8, 5935-5942.

(6) Long, R.; Liu, J.; Prezhdo, O. V., Unravelling the Effects of Grain Boundary and Chemical Doping on Electron-Hole Recombination in $\mathrm{Ch}_{3} \mathrm{nh}_{3} \mathrm{pbi}_{3}$ Perovskite by Time-Domain Atomistic Simulation. J. Am. Chem. Soc. 2016, 138, 3884-3890.

(7) Qiao, L.; Fang, W.-H.; Long, R.; Prezhdo, O. V., Extending Carrier Lifetimes in Lead Halide Perovskites with Alkali Metals by Passivating and Eliminating Halide Interstitial Defects. Angew. Chem. Int. Ed. 2020, 2020, 4684-4690.

(8) Perdew, J. P.; Zunger, A., Self-Interaction Correction to Density-Functional Approximations for Many-Electron Systems. Phys. Rev. B 1981, 23, 5048-5079.

(9) Even, J.; Pedesseau, L.; Jancu, J.-M.; Katan, C., Importance of Spin-Orbit Coupling in Hybrid Organic/Inorganic Perovskites for Photovoltaic Applications. J. Phys. Chem. Lett. 2013, 4, 2999-3005.

(10)Stoumpos, C. C.; Malliakas, C. D.; Kanatzidis, M. G., Semiconducting Tin and Lead Iodide Perovskites with Organic Cations: Phase Transitions, High Mobilities, and near-Infrared Photoluminescent Properties. Inorg. Chem. 2013, 52, 9019-9038. 
(11)Umari, P.; Mosconi, E.; De Angelis, F., Relativistic Gw Calculations on $\mathrm{Ch}_{3} \mathrm{nh}_{3} \mathrm{pbi}_{3}$ and $\mathrm{Ch}_{3} \mathrm{nh}_{3} \mathrm{sni}_{3}$ Perovskites for Solar Cell Applications. Sci. Rep 2014, 4, 4467.

(12)Jin, H.; Debroye, E.; Keshavarz, M.; Scheblykin, I. G.; Roeffaers, M. B. J.; Hofkens, J.; Steele, J. A., It's a Trap! On the Nature of Localised States and Charge Trapping in Lead Halide Perovskites. Mater. Horiz. 2020, 7, 397-410.

(13)Long, R.; Prezhdo, O. V., Dopants Control Electron-Hole Recombination at Perovskite$\mathrm{Tio}_{2}$ Interfaces: Ab Initio Time-Domain Study. Acs Nano 2015, 9, 11143-11155.

(14)Zhang, Z.; Long, R.; Tokina, M. V.; Prezhdo, O. V., Interplay between Localized and Free Charge Carriers Can Explain Hot Fluorescence in the $\mathrm{Ch}_{3} \mathrm{nh}_{3} \mathrm{pbbr}_{3}$ Perovskite: Time-Domain Ab Initio Analysis. J. Am. Chem. Soc. 2017, 139, 17327-17333.

(15)Long, R.; Fang, W.; Prezhdo, O. V., Moderate Humidity Delays Electron-Hole Recombination in Hybrid Organic-Inorganic Perovskites: Time-Domain Ab Initio Simulations Rationalize Experiments. J. Phys. Chem. Lett. 2016, 7, 3215-3222.

(16)Prezhdo, R. L. W.-H. F. O. V., Strong Interaction at the Perovskite/Tio 2 Interface Facilitates Ultrafast Photoinduced Charge Separation: A Nonadiabatic Molecular Dynamics Study. J. Phys. Chem. C 2017, 121, 3797-3806.

(17)Monkhorst, H. J. P., J. D., Special Points for Brillouin-Zone Integarations. Phys. Rev. B 1976, 13, 5188-5192.

(18)Akimov, A. V.; Prezhdo, O. V., Advanced Capabilities of the Pyxaid Program: Integration Schemes, Decoherence Effects, Multiexcitonic States, and Field-Matter Interaction. J. Chem. Theory Comput. 2014, 10, 789-804.

(19)Akimov, A. V.; Prezhdo, O. V., The Pyxaid Program for Non-Adiabatic Molecular Dynamics in Condensed Matter Systems. J. Chem. Theory Comput. 2013, 9, 4959-4972.

(20)Wang, Y.; Fang, W.-H.; Long, R.; Prezhdo, O. V., Symmetry Breaking at Mapbi Perovskite Grain Boundaries Suppresses Charge Recombination: Time-Domain Ab Initio Analysis. J. Phys. Chem. Lett. 2019, 10, 1617-1623.

(21)Quarti, C.; Mosconi, E.; De Angelis, F., Structural and Electronic Properties of OrganoHalide Hybrid Perovskites from Ab Initio Molecular Dynamics. Phys. Chem. Phys. Chem. 2015, 17, 9394-9409.

(22)Mukamel, S., Principles of Nonlinear Optical Spectroscopy. Oxford University Press: 1995.

(23)Kilina, S. V.; Neukirch, A. J.; Habenicht, B. F.; Kilin, D. S.; Prezhdo, O. V., Quantum Zeno Effect Rationalizes the Phonon Bottleneck in Semiconductor Quantum Dots. Phys. Rev. Lett. 2013, 110, 180404. 